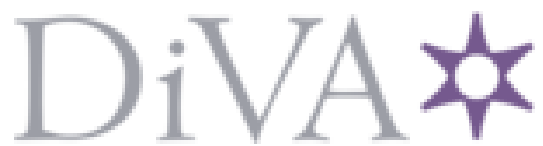

http://www.diva-portal.org

This is the published version of a paper published in Interacting with computers.

Citation for the original published paper (version of record):

Poole, E S., Comber, R., Hoonhout, J. (2015)

Disruption as a Research Method for Studying Technology Use in Homes

Interacting with computers, 27(1): 13-20

https://doi.org/10.1093/iwc/iwuo35

Access to the published version may require subscription.

N.B. When citing this work, cite the original published paper.

Permanent link to this version:

http://urn.kb.se/resolve?urn=urn:nbn:se:kth:diva-259238 


\title{
Disruption as a Research Method for Studying Technology Use in Homes
}

\author{
Erika S. PoOlE ${ }^{1, *}$, Rob COMBER ${ }^{2}$ AND JetTIE HoOnhout ${ }^{3}$ \\ ${ }^{1}$ College of Information Sciences and Technology, The Pennsylvania State University, 321 E IST Building, \\ University Park, PA 16802, USA \\ ${ }^{2}$ Culture Lab, Newcastle University, Newcastle upon Tyne, UK \\ ${ }^{3}$ Philips Research, Eindhoven, Netherlands \\ *Corresponding author: epoole@ist.psu.edu
}

\begin{abstract}
In this paper, we present disruption of household routines as a method to study home technology usage. Using three case studies as guidance, we show how disrupting household practices-either through changing the technology, task or division of labor-can provide valuable insight into current and future technology usage, and can guide the design of future technologies. Based on our case studies, we outline best practices and challenges with respect to the pragmatics of disruptive research methods.
\end{abstract}

\section{RESEARCH HIGHLIGHTS}

- We present disruption as a method for studying technology use in homes.

- Grounded in three case studies, we provide best practices for using this method.

- We address pragmatic and ethical challenges of using disruption as a method.

Keywords: HCI design and evaluation methods; user studies; interaction design process and methods; collaborative and social computing design and evaluation; ubiquitous and mobile computing design and evaluation; code of ethics

Received 21 January 2014; Revised 21 July 2014; Accepted 7 August 2014

\section{INTRODUCTION}

When developing human-centered technologies for the home, it is important to know about what people already $d o$ with respect to existing tasks and technologies. However, it can be difficult for people to articulate their needs, preferences and routines; many daily activities in the home are done in ways that do not invite conscious reflection (Crabtree and Rodden, 2004). For many years, the human-computer interaction (HCI) community has addressed the challenge of studying daily activities in the home using one or more of individual or group retrospective interviews, diary studies, ethnographic observations or probes. Although interviews and diaries are time-honored techniques in user experience research, they have marked limitations for studies of routines and coordination in homes. In particular, these techniques may lead to shallow accounting of technology interactions, particularly for studies of highly technical topics including a number of steps (Poole et al., 2009a). These techniques can also suffer from biases in which people, whether intentionally or unintentionally, incorrectly describe past events; while less discussed in HCI, this issue has been widely discussed in medical and legal communities (cf. Coughlin, 1990; Wells, 1993).

To address these challenges, HCI researchers have in many cases turned to probes. Probe-based approaches come in many forms, but in general can be characterized as items or activities intended to prompt reflection on aspects of daily life. Originally envisaged as ambiguous activities to prompt playful responses from participants (Gaver et al., 1999), probes have been adapted for a number of other purposes in HCI. These include using probes to as data collection technique, as a way to measure task performance, as a way to include users in the design process and as 'inspiration' for technology designers (Boehner et al., 2007). Not only have probes taken on a variety of purposes, they can be operationalized in a variety of forms and formats. For example, a common format is a package with a camera, a diary and cards with instructions, which are given to participants to document certain aspects of daily life or engage in creative ideation (Gaver et al., 1999; Mattelmäki, 2006). Probes can 
also be specially designed interactive technologies used in naturalistic settings- typically referred to as a technology probe (Hutchinson et al., 2003). Technology probes are not intended as prototype systems to be refined, but rather as 'throwaway technologies' used to explore the design possibilities for new technologies. What makes technology probes unique are that (i) they are inherently artificial-they are never intended to be commercially viable technologies; and (ii) they are inherently disruptive mechanisms that change ways in which people work and play, inviting reflection upon household norms. This disruptive nature of technology probes holds an especially useful benefit for researchers and product developers. Through disruption and change to normal routine, users may be able to more easily articulate key motivations and subjective qualities associated with routines; the use of technology probes can thus increase the usefulness of traditional HCI data collection mechanisms such as diaries, interviews or questionnaires.

Technology probes offer useful data. Nevertheless, they can also be expensive and time consuming to produce, with the fruits of extensive effort discarded at the end of the design ideation process. In this paper, we illustrate a complementary suite of technology probe-inspired disruptive techniques that, in many cases, can be less expensive and less time consuming across the lifespan of a project, while achieving similar results. For interaction design, disruption-oriented studies can offer valuable insights to better understand existing technologyrelated routines in homes (e.g. divisions of labor, values held by household members) as well as identify opportunities for the creation of future technologies.

Through three case studies, we illustrate the versatility of disruptive approaches for studying technology use in homes, and provide insight into best practices and challenges with respect to the pragmatics of disruptive research methods. The case studies include (i) the BinCam, a food preparation device for simplifying meal preparation; (ii) the Thermomix, a camera-augmented kitchen waste bin intended to change household waste practices; and (iii) the Home Network study, which asked householders to participate in updating and fixing networked information technology in their homes. Though all used a form of disruption as a research method, the motivations and mechanisms by which disruption occurred differed. One altered the technology, another altered the nature of the task and a third altered divisions of labor in completing tasks. Each provides insight into different ways in which disruption can be enacted as a home research technique, as well as challenges that may be encountered by researchers seeking to use disruptive methods in their own endeavors.

\section{CASE STUDY 1: DISRUPTION AS CHANGING THE TECHNOLOGY}

Our first case study centers on the broad problem of sustainability. Although many people hold positive attitudes toward environmental sustainability, there remain significant barriers to changing how individuals, households, communities and societies dispose of and recycle waste items. Given these barriers, it is not immediately clear what types of efforts can be taken at small-scale (e.g. at the individual or household level) to address this large-scale issue. The study we discuss here focuses on gaining a deeper understanding of sustainability behaviors in households, using disruption as a method. In this case study, the disruption was enacted by changing the technology used for disposing household waste. Specifically, households in this study used the BinCam (Comber and Thieme, 2013; Thieme et al., 2012). The BinCam (Fig. 1) used a commercially available kitchen refuse bin, but added capability to automatically log disposal of items through digital images captured by a smartphone installed on the underside of the bin lid. Captured images were uploaded to an application on Facebook. Here, users could explore, review or share communications about waste-related behaviors of themselves and others. Thus, the nature of the task (waste disposal) was not changed, but rather the technology was subtly altered to promote reflection and social comparison.

The study population consisted of university student households; students are a population known to be under-aware of the implications of inappropriate waste behavior (WRAP, 2010). At the same time, as some of the most prolific users of social media, students living in shared apartment housing are a population for which we can enmesh and collide that which participants do not care about (recycling), with that which they do (their social lives). While primarily designed to 'change' behavior, the BinCam system was built on the premise of

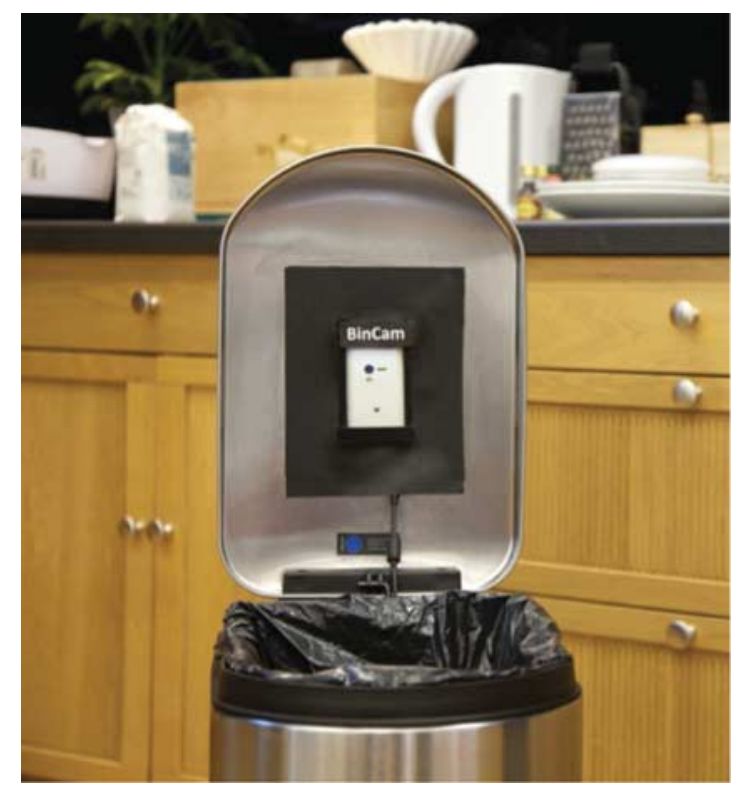

Figure 1. The initial BinCam system with a mobile phone in the lid of the bin. The presence of a familiar device, and its functionality, in an unexpected location disrupts conventions for waste behavior. 
increased self-awareness and reflection. As such, it constituted a key data source in reconstructing everyday practices with participants, both directly as a prompt in post-study interviews and indirectly as an anchor for reflecting on personal action during the study.

The presence of the bin with a camera in the lid and the presence of photographs of waste in a Facebook stream both served to disrupt normative behavior and assumptions. BinCam created visibility of waste behavior by connecting separate physical and social spaces (Ganglbauer et al., 2012) and by encouraging participants to visit and later revisit the invisible practices of waste in settings where it is expected (kitchen) and unexpected (social media).

\subsection{What did changing the technology reveal?}

While the BinCam system was designed to be a provocative system, in its first studies one of the most disruptive mechanisms was not a 'designed' feature but an artifact of the mobile phone used to take photos (Thieme et al., 2012). Whenever the mobile phone attached to the bin lid took a picture, it emitted a simulated shutter noise. This noise occurred at the moment when food waste was best ignored-just after the disposalbut was powerful in drawing attention and reflection onto that act. In later studies, this audio disruption became a focus for this stream of research, revealing much about the unconscious habits of food waste (Comber and Thieme, 2013). The noise simultaneously drew attention to the complex contexts in which we deploy interactive systems and the inevitable difficulty in addressing this problem (cf. Bardzell et al., 2012). It further connected the integrated and distributed practices through which waste is produced (Ganglbauer et al., 2013).

\section{CASE STUDY 2: DISRUPTION AS CHANGING THE TASK}

In our second case study, we examine home appliance design, and demonstrate a second form of disruption: changing the task that household members complete. When developing a revision of an appliance-for instance a washing machine or a blender-it can be relatively easy to determine what needs to be done to improve upon existing technologies. One could, for example, start with observing consumers while using current products, and involve them in discussions about what could be improved (e.g. Beyer and Holtzblatt, 1997). Alternatively, one could examine features of competing products, or review online customer reviews or technical support requests about the product. How, though, can user experience be accounted for in product concepts conceived from 'scratch'?

One of the authors of this article faced exactly this issue when exploring whether and how novel technologies-kitchen appliances that had not yet been invented - could improve home food preparation experiences.
Every day, people prepare and consume food. These activities can be special (e.g. preparing an extravagant holiday meal) but more often are mundane and routine in nature. When interviewing informants about such everyday routines, it often turns out to be hard for them to express the methods and motivations for carrying out such activities (Comber et al., 2013; Hoonhout, 2007; Hoonhout et al., 2014). With respect to the topic of food preparation, informants are easily able to discuss considerations such as preparation time, healthiness and taste of foods. But certain routine, and unremarkable elements in the actual food preparation experience may not come to mind so easily; that being said, these less tangible elements of experience can impact consumer reception to new technologies (Norman, 2007).

Thus, the aim of the second case study was to get more indepth insight into subjective aspects of cooking experience, through changing the nature of the cooking task itself. A commercially available, but uncommon food preparation appliance - the Thermomix — served as a way to disrupt the task of food preparation. The Thermomix (see Fig. 2) is not like any other kitchen appliance, and it requires users to prepare meals in a very different way. The device can chop, stir and heat at the same time. It monitors the heating process, and automatically adjusts when required. It inherently changes both the tasks of food preparation, and the technologies used to complete these tasks. For example, given the chopping knives, the system employs safety guards, preventing users from easily opening the lid during the cooking process.

In a field trial with 10 homes that had never used the device before, the participants were asked to use the Thermomix for

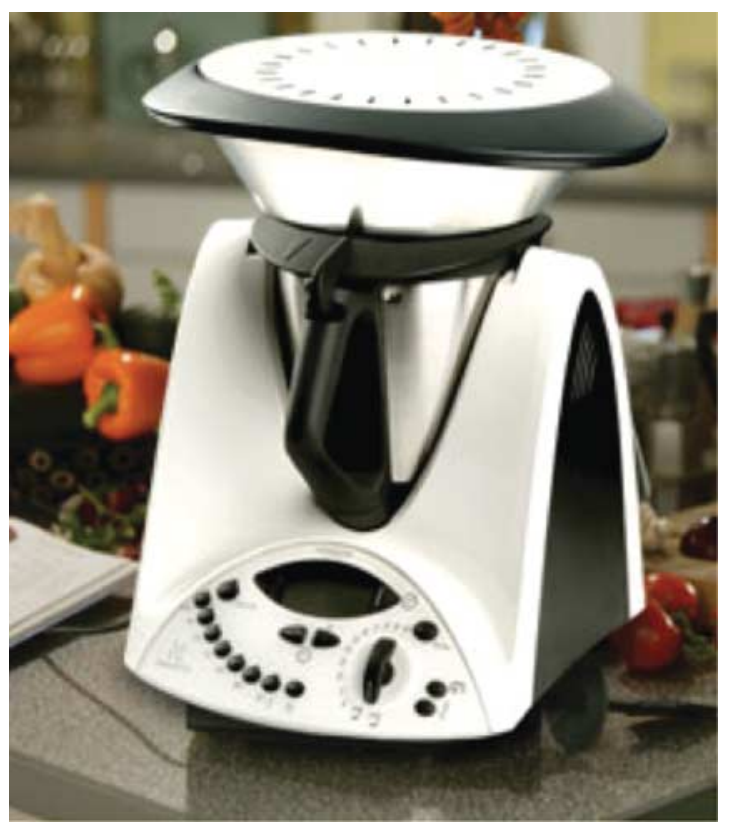

Figure 2. The Thermomix device automates food preparation tasks, including food weighing, chopping, stirring and heating. http://www. thermomix.com. 
3-6 weeks and record their experiences using diaries, photographs, and interviews during and after the test period. Using this new, unknown device triggered the participants to think about what sort of devices they would like to use in the future: what sort of features, functionalities and qualities should such devices should have? How might they use these devices in their daily life?

In comparison to prior interview-only studies, having study participants also use a Thermomix in home over an extended period of time created a richness that was not seen when only using interviews. Specifically, this approach helped participants articulate certain aspects of daily cooking activities that were very dear to them, but not consciously thought about until the cooking process was made different. In summary, participants expressed aspects in food preparation that they found very important, that we did not hear so strongly, or did not even hear at all from interviewees, in (interview) studies we had conducted before.

\subsection{What did changing the task reveal?}

Participants who used the Thermomix remarked on the importance of having control over the results; that is, being the 'master of the cooking process'. Being in charge of the cooking process meant being able to monitor the process through stirring, smelling and looking - all things which the Thermomix does not allow so easily. We did not anticipate, and indeed found it surprising, that participants would long for the simple act of stirring a pot. At the same time, participants also remarked on the benefits of 'unattended' cooking, especially during hectic situations families may experience around meal preparation times. Thus, this might suggest that future appliances provide flexibility with respect to whether control of the cooking process should go to the machine or the person.

\section{CASE STUDY 3: DISRUPTION AS CHANGING THE DIVISION OF LABOR}

In the third case study, which focused on user experience difficulties associated with home network device installation, configuration and troubleshooting, neither the tasks nor the technologies substantially changed. Instead, the household division of labor in completing technology-related tasks was changed from the norm. This was done in order to learn more about household norms associated with fixing and seeking help for technology problems that may occur in homes, which prior studies identified as a potential barrier (Poole et al., 2009b).

Our ultimate goal in this study was to inform the design of future tools for technical assistance in residential settings. While HCI has ample advice on how to design quality user experiences on desktop and mobile computing devices, networked technologies present additional challenges. Specifically, as technologies in the home move from single-user, standalone devices to networked technologies, end users who are tasked with setting up, maintaining, and troubleshooting information technologies may now interact not just with end user devices but also with infrastructures (e.g. home networks) that have no clean and clear interfaces for use (Edwards and Grinter, 2001; Grinter et al., 2005). Yet studying how users interact with these distributed, interfaceless devices can be challenging. Even more so, studying how users react to and recover from infrastructural level technology problems calls for new methodological approaches. Traditional techniques for assessing usability may not be appropriate when used in the context of studying devices and interconnections associated with home networks.

For instance, depending on the research questions at hand, one could use lab experiments to observe troubleshooting practices in situ. The tradeoff, however, is the absence of richness that occurs in real-life settings with respect to troubleshooting and configuring technologies; networked home infrastructures involve a number of stakeholders, and the knowledge and ability to make changes is spread across these stakeholders, creating problems of dependencies. Overall, studying household coordination and divisions of labor unfolding over time and space is not something easily addressed in a laboratory environment. To close this gap, disruption of routines through changing the person completing a task in a naturalistic environment can serve as a vital component in the home technology research methods toolbox.

Thus, in this case study, household members were asked to interact with home technological infrastructures in ways that potentially disrupted the social norms of the home (Poole, 2012). For example, all members of a home enrolled in the study were asked to participate in a series of home technology installation, maintenance and teaching practices, even if they did not normally do so outside of the confines of the study.

\subsection{What did changing the division of labor reveal?}

Shifting installation and configuration responsibilities to another person-even for just a week at a time-allowed for deeper understandings of labor divisions in homes. For instance, people who lived in houses where an occupant had a small business relying on Internet connectivity were far more cautious about installing new devices or making network configuration changes that could interrupt business workflow. It also provided fodder for discussion about power dynamics between more and less technically skilled household members; we found that people wanted to learn more about how to participate in technology-related tasks, or make decisions about how and why things were configured within the home, but were excluded from these processes outside the confines of the study. Ultimately, by disrupting who completed the task, we identified aspects of social context that may be important to consider in future technical assistance tools, as well as barriers for individuals who want to learn about information technologies in informal (non-school) settings. 


\section{DISCUSSION: THE PRAGMATICS OF DISRUPTION AS RESEARCH METHOD}

The reported case studies all used some form of disruption, which provided insights into people's behavior with everyday activities, tasks and technologies. However, given the potential impact of disruptions on daily life and routines, this approach is not one to adopt lightly. In the following sections, we outline several challenges encountered in our experiences using disruption as a research technique. While many of these challenges are universal to research involving human subjects, they are particularly pronounced in studies involving disruption.

\subsection{Challenge \#1: Researcher authority and participant discomfort}

Studies relying on disruption are challenging because they are by their very nature disruptive, and can be unpleasant to participants or other people in their households or local community. As researchers, it is important to provide ways to increase participant comfort with the activities, and not feel overly pressured to participate. Participants might see the researcher as an authority to whom they feel obliged to comply, an issue long discussed in social sciences research (Milgram, 1963) and more recently in HCI (Dell et al., 2012). This challenge requires careful consideration in instructing the participants, informing them about their rights as a research subject, and cultivating environments that encourage the participants to speak up about their true desires, even if those desires are disappointing to the research team.

In the Thermomix study, the research team addressed issues of participant comfort by having discussions with the participants the length of time that the device would be in their possession, and the number of times that would be fine with them to actually prepare a meal with it (or a meal component, e.g. soup, or a dessert, not the complete meal). This was done to make sure that they would not feel coerced to have to use the device everyday, or for every meal.

The Home Network study faced similar challenges, but took a different approach. Inherently, the study was designed to put participants into roles that could be uncomfortable. Participants could, by the nature of the study, look unknowledgeable or foolish, or cause problems that limit the ability of other people in the home to use a shared resource (e.g. the home network). In other words, there were very clear disincentives to participating in the study. To overcome this challenge, participant gratuities were linked to attempting (but not necessarily completing) a percentage of home network-related tasks from a pool of available choices. We thought that allowing some flexibility in which tasks were chosen would make participants feel more comfortable with participating in the study. For some households, particularly those with occupants who enjoyed or at least tolerated new technology, this approach worked.
However, we did not fully appreciate how overwhelming some of the tasks would appear to people who were technology averse. Furthermore, it is possible that this accidentally created a coercive situation for participants: they wanted to receive the gratuities, but at the same time, may have felt pressure to complete the unwanted tasks in order to receive payment. Based on this experience, we recommend any of the following options: (i) the researcher and participant co-operatively undertake the first few tasks as a team; (ii) the researcher and participant collectively design the tasks; or (iii) use behavioral sciences techniques such as motivational interviewing (Miller and Rose, 2009) to cooperatively decide pros and cons and what is appropriate in the course of the study.

\subsection{Challenge \#2: Researcher-participant relationships can threaten validity}

The close relationship between researcher and participant resulting from the combination of having technologies installed in family homes and longer-term trials can introduce social desirability biases. For instance, participants may want to please, or at least avoid disappointing the researcher. This may lead to participants working with the technology in the study even if they no longer feel comfortable doing so. In fact, one of the authors worked with a household that earnestly asked at every visit whether they were 'good participants' and wanted to know about how well (or poorly) other homes fared.

Similarly, projects in which participants and researchers work collaboratively toward understandings of technology use can blur the boundary between researcher and the researched, turning participants into peers and collaborators. The close relationship that can develop can result in biases in the results that are being collected; for instance, a participant's investment in co-creating a technology might result in a positive bias toward the artifact that may not be seen when the technology is scaled to a more widespread audience.

\subsection{Challenge \#3: Biases and novelty effects of disruption}

There is a risk that researchers may report elements that are an artifact of a disruptive probe, rather than being linked to the routine activity itself. In work complementary to our own case studies, Tolmie and Crabtree (2008) discussed how home technology research deployments of any sort could be considered breaching experiments in their analysis of Gaver's (2006) Video Window. That is, introducing an alien artifact into a home might result in bias simply due to the presence of the tool. This is a widely recognized problem in experimental design, yet in the case of HCI research in the home this concern is often ignored. This may be due to the unwritten understanding of 'interventionist' HCI studies-that is, when introducing new systems or when engaging with participants-we openly 
acknowledge that our results may be open to biases, novelty effects, and the socially desirable responses from participants. Even so, we must acknowledge limitations in understanding the naturalistic context of the home. In addition, there is a need for sensitive selection and design of suitable disruptive tasks, technologies and labor divisions. A carefully constructed test protocol, proper understanding of the disruptive probe's qualities, a detailed post-test interview, and solid analysis of the data can further help to enrich that understanding.

\subsection{Challenge \#4: Disruptions can impact participant self-identity}

When comparing the three case studies, each has a different potential for impacting individual and collective norms of the home. The Thermomix, as a new device for food preparation, did not inherently invite negative scrutiny from the user's family, friends or acquaintances. On the other hand, BinCam had potential to publicly broadcast people defying desirable social norms such as discarding recyclables improperly or wasting uneaten foods. The Home Network study also offered possibilities of exposing lack of knowledge, or other undesirable perceptions of participants.

Disruption confronts the participant with both their assumptions and the conventions with which they construct their self-identities and their homes. With BinCam, this confrontation was reflected on by participants as producing feelings of guilt. Although designed to understand and support positive behavior change, the disruption implies a negative appraisal of existing practices. This has significant implications for the emotional wellbeing of participants and for their performance of identity, as the work of home life may be catapulted into a national arena. In the case of BinCam, private moments become open to public scrutiny. In response to increased scrutiny and peer surveillance about waste habits, student households participating in the study found new and social ways to reduce waste, such as sharing large meals and purchasing food together. Although a positive outcome in this regard, use of disruption that can invite negative social appraisal can have the potential to change social relationships in the home, and not always for the better.

As researchers and practitioners, we must therefore devise clear strategies for the safeguarding of participant wellbeing. For instance, in the Home Network study, participants were informed at the beginning of the study of what they would be doing, that participation was voluntary, and that it was possible that they could inflict temporary damage on their home networks. To address these risks, if participants were stuck on a given task, a member of the research team would make an emergency house call to cooperatively troubleshoot the issue and, in a sense, 'talk the participant down from the ledge'. For the majority of households, this approach worked well. In fact, we had instances in which participants reported that they felt empowered and proud of being more technically capable than they had imagined.

\subsection{Challenge \#5: Disruptions within the home can ripple beyond the home}

While this paper discusses methods for research within the home, the defining boundaries of the home are a continued source of debate. Although the home could be considered to be bounded by four walls, and in many contexts this is appropriate, a simple look at the technologies of the home, such as TV, telephone, personal computer, mobile phone and so on, suggest that many of the activities of the home transcend the boundaries of the 'house as home'. Furthermore, homes are open not just to the core inhabitants, but also to family, friends and other visitors who might stay for various lengths of time. Such visits form a special ethical problem - whereas participants will have been informed and provided with instructions, know about the type of information that is being collected, and are prepared to interact with a device, this will not be the case for the visitors.

The relationships that are expressed in and through the home are fundamental to our personal and social identity beyond the home. It is therefore crucial to understand the implications of disruptive technology to ripple beyond the site of research. In the case of the Thermomix, this problem was minimized. The device was also of a size that it could be stored in a private location while visitors were in the home, avoiding questions or scrutiny. Furthermore, through the way the study was designed, participants could simply choose not to use the device in the company of others, so as to avoid serving guests unsavory meals (which was a reported issue raised by study participants). The BinCam, however, was not hidden from guests, and could inadvertently capture and share the waste disposal practices of visitors without permission. Even for the people living in the home, the BinCam system, with its connection to social media, had the potential to disrupt conventions of privacy around household waste, access to personal and intimate behaviors and understandings of space. Simply, BinCam was a boundarycrossing object from its inception.

As a provocative system, BinCam drew attention from the mass media (see Vines et al., 2013 for an in depth discussion of the implications). What became clear was that, although designed ostensibly to promote reflection on household recycling behavior, the BinCam system promoted wider reflection among the public on topics of sustainability, privacy, politics and the role of technology in our everyday lives. As such, the disruptive technology system can be understood to connect across different scales of action (Dourish, 2010).

Analysis of the effect of mass media on HCI research (Vines et al., 2013) and analysis of the deployment of ubiquitous technologies in the home (Edwards and Grinter, 2001) suggest that the ripple effect of domestic technologies can change the national and international debate on issues such as sustainability, the doing of work in the home and the 
moral rightness of caregiving in the home, such as parenting. Such impact on societal debate can be understood through the continuous contestation in and through design that provokes insight on the power relations of social contexts, technology design and technology use (DiSalvo, 2012). Intentional disruption is one mode of agonism - regardless of its application to technology design or social systems.

\section{CONCLUSION}

In designing disruption-oriented studies, we have demonstrated the opportunities afforded by disrupting divisions of labor, tasks and technologies used. Despite the attractiveness of this approach, we must carefully consider whether disruption is the most suitable way to achieve an end goal a researcher may have. Disruption offers rich insights, but can also have pragmatic and ethical challenges. In some cases, disruption may be the best possible choice. For instance in the case of the Home Network study, there are few other options to studying issues associated with household division of technical labor. Yet, in other projects, it may be better to use alternative means of data collection. Many methods are available. For instance scenarios, which are short illustrated stories of how a device might be used, could help to explain the potential impact of new technology (Carroll, 2000). When discussing such scenarios with users, they will get a general idea of a new concept; but since it is still a rather abstract representation, users might again not find it easy to appreciate how mundane routines will be impacted. Tailored scenarios may address some of these issues, but also offer challenges (Coughlan et al., 2013). In addition, user enactment techniques, in which participants respond to storyboards of technology design ideas, roleplaying and re-enactments of their day-to-day routines in the presence of the researchers, may also provide insight into finer aspects of technology design for homes (Odom et al., 2012). That being said, scenarios and user enactments offer distant and short interaction opportunities, which may not provide the same level of richness as an extended in situ study using disruption as a method.

Overall, the use of disruption as a research method in the home provides unique insights on the routines, values and practices of members of the household. In our three case studies, disruption serves to provide a new lens on routine behavior, prompt reflections on assumptions about normative behavior, connect individual behavior to the wider context, and to prompt further consideration of the role of relationships in the work of the home with relation to technology. Yet, disruption brings with it a number of practical and ethical concerns. Our case studies highlight issues of obedience, affective disruption and mending, and the potential for disruptive methods to ripple beyond the home. While disruption will continue to be a useful method, we urge fellow researchers following lessons learned to carefully unpick the implications of disruption for the social, technical and political context of technology use in the home.

\section{ACKNOWLEDGEMENTS}

The BinCam project has been supported by the EU FP7 Balance@Home project and the RCUK Research Hub on Social Inclusion through the Digital Economy (SiDE). The Home Network project was supported by an NSF graduate research fellowship.

\section{REFERENCES}

Bardzell, S., Bardzell, J., Forlizzi, J., Zimmerman, J. and Antanitis, J. (2012) Critical Design and Critical Theory: The Challenge of Designing for Provocation. Proc. Designing Interactive Systems Conf., Newcastle Upon Tyne, UK, pp. 288-297. ACM.

Beyer, H. and Holtzblatt, K. (1997) Contextual Design: Defining Customer-Centered Systems. Elsevier, Amsterdam.

Boehner, K., Vertesi, J., Sengers, P. and Dourish, P. (2007) How HCI Interprets the Probes. Proc. SIGCHI Conf. on Human Factors in Computing Systems, San Jose, CA, USA, pp. 1077-1086. ACM.

Carroll, J.M. (2000) Making Use: Scenario-Based Design of HumanComputer Interactions. MIT Press, Cambridge, MA.

Comber, R. and Thieme, A. (2013) Designing beyond habit: opening space for improved recycling and food waste behaviors through processes of persuasion, social influence and aversive affect. Pers. Ubiquitous Comput., 17, 1197-1210.

Comber, R., Hoonhout, J., Van Halteren, A., Moynihan, P. and Olivier, P. (2013) Food Practices as Situated Action: Exploring and Designing for Everyday Food Practices with Households. Proc. SIGCHI Conf. on Human Factors in Computing Systems, Paris, France, pp. 2457-2466. ACM.

Coughlan, T., Brown, M., Lawson, G., Mortier, R., Houghton, R. J. and Goulden, M. (2013) Tailored Scenarios: A Low-Cost Online Method to Elicit Perceptions on Designs Using Real Relationships. CHI'13 Extended Abstracts on Human Factors in Computing Systems, Paris, France, pp. 343-348. ACM.

Coughlin, S.S. (1990) Recall bias in epidemiologic studies. J. Clin. Epidemiol., 43, 87-91.

Crabtree, A. and Rodden, T. (2004) Domestic routines and design for the home. Comput. Support. Coop. Work, 13, 191-220.

Dell, N., Vaidyanathan, V., Medhi, I., Cutrell, E. and Thies, W. (2012) Yours is Better!: Participant Response Bias in HCI. Proc. SIGCHI Conf. on Human Factors in Computing Systems, Austin, TX, USA, pp. 1321-1330. ACM.

DiSalvo, C. (2012) Adversarial Design. The MIT Press, Cambridge, MA.

Dourish, P. (2010) HCI and Environmental Sustainability: The Politics of Design and the Design of Politics. Proc. 8th ACM Conf. on Designing Interactive Systems, Aarhus, Denmark, pp. 1-10. ACM.

Edwards, W.K. and Grinter, R.E. (2001) At Home with Ubiquitous Computing: Seven Challenges. Ubicomp 2001: Ubiquitous Computing, pp. 256-272. Springer, Berlin, Heidelberg.

Ganglbauer, E., Fitzpatrick, G. and Molzer, G. (2012) Creating Visibility: Understanding the Design Space for Food Waste. Proc. 
11th Int. Conf. on Mobile and Ubiquitous Multimedia, Ulm, Germany, p. 1. ACM.

Ganglbauer, E., Fitzpatrick, G. and Comber, R. (2013) Negotiating food waste: using a practice lens to inform design. ACM Trans. Comput.-Hum. Interact., 20, 11.

Gaver, W.W. (2006) The video window: my life with a ludic system. Pers. Ubiquitous Comput., 10, 60-65.

Gaver, B., Dunne, T. and Pacenti, E. (1999) Design: cultural probes. Interactions, 6, 21-29.

Grinter, R.E., Edwards, W.K., Newman, M.W. and Ducheneaut, N. (2005) The Work to Make a Home Network Work. ECSCW 2005, pp. 469-488. Springer, Netherlands.

Hoonhout, H.C.M. (2007) Setting the stage for developing innovative product concepts: people and climate. CoDesign, 3, 19-34.

Hoonhout, J., Gros, N., Geleijnse, G., Nachtigall, P. and van Halteren, A. (2014) What are We Going to Eat Today? Food Recommendations Made Easy and Healthy. In Choi, J.H.J., Foth, M. and Hearn, G. (eds) Eat, Cook, Grow: Mixing Human-Computer Interactions with Human-Food Interactions, pp. 51-64. MIT Press, Cambridge, MA.

Hutchinson, H. et al. (2003). Technology Probes: Inspiring Design for and with Families. Proc.of the SIGCHI Conf., on Human Factors in Computing Systems, pp. 17-24.

Mattelmäki, T. (2006). Design Probes. University of Art and Design, Helsinki, Finland.

Milgram, S. (1963) Behavioral study of obedience. J. Abnormal Soc. Psychol., 67, 371.

Miller, W.R. and Rose, G.S. (2009) Toward a theory of motivational interviewing. American Psychologist, 64, 527-573.

Norman, D.A. (2007) Emotional Design: Why We Love (or Hate) Everyday Things. Basic Books, New York.
Odom, W., Zimmerman, J., Davidoff, S., Forlizzi, J., Dey, A.K. and Lee, M.K. (2012) A Fieldwork of the Future with User Enactments. Proc. Designing Interactive Systems Conf., Newcastle Upon Tyne, UK, pp. 338-347. ACM.

Poole, E.S. (2012) Interacting with Infrastructure: A Case for Breaching Experiments in Home Computing Research. Proc. ACM 2012 Conf. on Computer Supported Cooperative Work, Seattle, Washington, pp. 759-768. ACM.

Poole, E.S., Edwards, W.K. and Jarvis, L. (2009a) The home network as a socio-technical system: understanding the challenges of remote home network problem diagnosis. Computer Support. Coop. Work, 18, 277-299.

Poole, E.S., Chetty, M., Morgan, T., Grinter, R.E. and Edwards, W.K. (2009b) Computer Help at Home: Methods and Motivations for Informal Technical Support. Proc. SIGCHI Conf. on Human Factors in Computing Systems, Boston, USA, pp. 739-748. ACM.

Thieme, A., Comber, R., Miebach, J., Weeden, J., Kraemer, N., Lawson, S. and Olivier, P. (2012) We've Bin Watching You: Designing for Reflection and Social Persuasion to Promote Sustainable Lifestyles. Proc. SIGCHI Conf. on Human Factors in Computing Systems, Austin, TX, USA, pp. 2337-2346) ACM.

Tolmie, P. and Crabtree, A. (2008) Deploying Research Technology in the Home. Proc. 2008 ACM Conf. on Computer Supported Cooperative Work, San Diego, CA, USA, pp. 639-648. ACM.

WRAP (2010) Waste and resources action programme. Retrieved from http://www.wrap.org.uk (accessed November 3, 2014).

Wells, G.L. (1993) What do we know about eyewitness identification? Am. Psychol., 48, 553.

Vines, J., Thieme, A., Comber, R., Blythe, M., Wright, P.C. and Olivier, P. (2013) HCI in the Press: Online Public Reactions to Mass Media Portrayals of HCI Research. Proc. SIGCHI Conf. on Human Factors in Computing Systems, Paris, France, pp. 1873-1882. ACM. 